\title{
Ellipse packing in two-dimensional cell tessellation: A theoretical explanation for Lewis's law and Aboav-Weaire's law
}

\author{
Kai Xu ${ }^{\text {Corresp. } 1}$ \\ ${ }^{1}$ Fisheries College, Jimei University, Xiamen, China \\ Corresponding Author: Kai Xu \\ Email address: kaixu@jmu.edu.cn
}

Background: Lewis's law and Aboav-Weaire's law are two fundamental laws used to describe the topology of two-dimensional (2D) structures; however, their theoretical bases remain unclear.

Methods: We used software R with package Conicfit to fit ellipses based on the geometric parameters of polygonal cells of ten different kinds of natural and artificial 2D structures.

Results: Our results indicated that the cells could be classified as an ellipse's inscribed polygon (EIP) and that they tended to form the ellipse's maximal inscribed polygon (EMIP). This phenomenon was named as ellipse packing. On the basis of the number of cell edges, cell area, and semi-axes of fitted ellipses, we derived and verified new relations of Lewis's law and Aboav-Weaire's law .

Conclusions: Ellipse packing is a short-range order that places restrictions on the cell topology and growth pattern. Lewis's law and Aboav-Weaire's law mainly reflect the effect of deformation from circle to ellipse on cell area and the edge number of neighboring cells, respectively. The results of this study could be used to simulate the dynamics of cell topology during growth. 
1 Ellipse packing in two-dimensional cell tessellation: A theoretical explanation for Lewis's law and Aboav-

\section{Weaire's law}

3

4 Kai Xu

5 Fisheries College, Jimei University, Xiamen, 361021, China

$6 \quad$ Email: kaixu@jmu.edu.cn

7 


\section{Abstract}

9 Background: Lewis's law and Aboav-Weaire's law are two fundamental laws used to describe the topology of two10 dimensional (2D) structures; however, their theoretical bases remain unclear.

12 Methods: We used software R with package Conicfit to fit ellipses based on the geometric parameters of polygonal cells of ten different kinds of natural and artificial 2D structures.

15 Results: Our results indicated that the cells could be classified as an ellipse's inscribed polygon (EIP) and that they tended to form the ellipse's maximal inscribed polygon (EMIP). This phenomenon was named as ellipse packing. On the basis of the number of cell edges, cell area, and semi-axes of fitted ellipses, we derived and verified new relations of Lewis's law and Aboav-Weaire's law.

Conclusions: Ellipse packing is a short-range order that places restrictions on the cell topology and growth pattern. of cell topology during growth. 


\section{Introduction}

A two-dimensional (2D) plane can be tessellated by convex polygons. Scientists are interested in natural and artificial 2D structures that share the common feature that the coordination number of vertices (the number of edges meeting at a vertex) of polygonal cells always equals three. The cell topology of these $2 \mathrm{D}$ structures can be described according to three laws: Euler's law, Lewis's law, and Aboav-Weaire's law (Weaire \& Rivier 1984). The latter two laws were first observed empirically by Lewis and Aboav, with the original aims of understanding laws in biological and physical structures, respectively (Aboav 1970; Lewis 1926; Lewis 1928; Weaire 1974). Although Lewis's law and Aboav-Weaire's law are essential for understanding the formation mechanisms of 2D structures, their theoretical explanations are deficient (Mason et al. 2012; Weaire \& Rivier 1984). The coordination number is a short-range order that mathematically determined that the average number of edges per cell is six (Graustein 1931).

When this study restricts attention to biological 2D structures, the word "cell" represents the top and bottom faces of a prismatic cell. The dynamics of cell topology during growth make biological 2D structures even more complicated than other types of 2D structures. For example, internal angles of Pyropia haitanensis cells have been concentrated in the range of $100-140^{\circ}$ by direction-specific division and direction turning of cell edges, which suggested that the cells tended to form regular polygons (Xu et al. 2017). These observations hinted at the possibility of undiscovered short-range orders in 2D structures. A recent study by Xu et al. (2018) found that the effective coverage area of ellipse-shaped exoskeletons of microalga Emiliania huxleyi cells tended to approach the maximal area of an ellipse's inscribed polygon (EIP). This study identified a similar phenomenon: the polygonal cells of natural and artificial 2D structures were inclined to form the ellipse's maximal inscribed polygon (EMIP). On the basis of this short-range order, the present study derived and verified new relations of Lewis's law and AboavWeaire's law.

\section{Materials and methods}

We used Amscope Toupview 3.0 software to analyze the images of ten kinds of 2D structures. The images of $P$. haitanensis and two images of onion were taken by the author of this study, and the others were derived from the published papers. The nonliving biological 2D structures included the following: cross-sections of shells of Atrina rigida, Atrina vexillum, and Pinna nobilis (Reich et al. 2018). The living biological 2D structures included the following: epidermal tissues of Agave attenuate, Allium cepa (onion), and Allium sativum (garlic) (Mombach et al. 
53

1990); and P. haitanensis thalli. The physical 2D structures include amorphous silicon dioxide $\left(\mathrm{SiO}_{2}\right)$ film (Büchner et al. 2016) and soap (Aboav 1980). The random-seeded Voronoi diagrams are artificial 2D structures that also have been used for analysis (Aboav 1985). For each polygonal cell, we measured the area $\left(\mathrm{A}_{C}\right)$, coordinates of center $\left(\mathrm{X}_{P C}\right.$ , $\left.\mathrm{Y}_{P C}\right)$, and vertices $\left(\mathrm{X}_{V}, \mathrm{Y}_{V}\right)$. We used software $\mathrm{R}$ (version 3.5.1) with package Conicfit to fit an ellipse based on the coordinates of the vertices of each polygonal cell (Fig. 1A) (Chernov et al. 2014). For the $\mathrm{SiO}_{2}$ film, the vertices of polygonal cells were formed by the silicon $(\mathrm{Si})$ atoms. Five geometric parameters could be used to describe the ellipse, which include the semi-major axis $a$, semi-minor axis $b$, coordinates of center $\left(\mathrm{X}_{E C}, \mathrm{Y}_{E C}\right)$, and angle of tilt of the major axis $\theta$ (Fig. 1B). On 2D geometry, five points determine a conic, for example, the ellipse. For polygons with five or more edges, we set $\mathrm{X}_{P C}$ and $\mathrm{Y}_{P C}$ as the initial values of the coordinates of the ellipse center to improve fitting. As for cells with only four edges, we combined the coordinates of the four vertices and the four midpoints of the edges as a single data set to fit an ellipse in the same manner as the cells with five or more edges. Then, we set the geometric parameters of the fitted ellipse as the initial values to fit the second ellipse for the coordinates of the four vertices. We found the second ellipse to be the smallest one among all of the fitted ellipses, and which we used for analysis. We provide our reasons for finding the smallest circumscribed ellipses for four-edged polygonal cells in the next section.

We calculated the area of the ellipse $\left(\mathrm{A}_{E}\right)$ as follows:

$$
\mathrm{A}_{E}=\pi a b
$$

The area of the maximal inscribed polygon of the ellipse $\left(\mathrm{A}_{M I P}\right)$ is

$$
\mathrm{A}_{M I P}=0.5 \operatorname{nabsin}\left(\frac{2 \pi}{n}\right),
$$

where $n$ is the number of edges of inscribed polygon ( $\mathrm{Su} 1987)$. The form deviation of vertex (FD) is

$$
F D=\frac{\mathrm{D}_{V C}-R}{R} \times 100 \%,
$$

where $\mathrm{D}_{V C}$ is the distance between a vertex and the center of the fitted ellipse (the length of line VC)

$$
\mathrm{D}_{V C}=\sqrt{\left(\mathrm{X}_{V}-\mathrm{X}_{E C}\right)^{2}+\left(\mathrm{Y}_{V}-\mathrm{Y}_{E C}\right)^{2}}
$$

$R$ is the distance from the ellipse center to the cross point of the fitted ellipse and the line $\mathrm{VC}$

$$
R=\frac{a b}{\sqrt{(\operatorname{asin}(\arctan (\tan \theta)-\delta))^{2}+(b \cos (\arctan (\tan \theta)-\delta))^{2}}},
$$

where $\delta$ is the angle between line $\mathrm{VC}$ and $\mathrm{X}$-axis, the ranges of $\theta$ and $\delta$ are $[0, \pi)$ and $(-0.5 \pi, 0.5 \pi)$, respectively 
(Fig. 1B). R code (Document S1) and three examples (Dataset S1) for these calculations are included in the supplementary files.

\section{Results and discussion}

\section{Ellipse packing}

Thallus of red alga $P$. haitanensis is a single-layered prismatic cell sheet that is a mathematical consequence of 2D expansion on a plane by cell proliferation (Xu et al. 2017). Thus, $P$. haitanensis thalli can be simplified as 2D structures. We found the average number of edges of $P$. haitanensis cells to be $6.0 \pm 0.9$ (1375 cells in 13 thalli were examined; Table 1), which was consistent with previous studies on P. haitanensis as well as studies on many other organisms and physical structures (Gibson et al. 2006; Sánchez-Gutiérrez et al. 2016; Weaire \& Rivier 1984; Xu et al. 2017). According to Euler's 2D formula, this kind of phenomenon has been mathematically determined when the coordination number of each vertex equals three when different-size cells tessellate a 2D plane (Graustein 1931; Weaire \& Rivier 1984). The size differences between cells indicated that these 2D structures display a long-range disorder, because the unit cell has neither periodicity nor translational symmetry. In addition, the average number of edges of $P$. haitanensis cells quickly approached six with an exponential increase in cell number resulting from an increase in body size (Xu et al. 2017). Thus, this phenomenon has been observed only when the 2D structures contain a large number of cells (Graustein 1931; Lewis 1926; Weaire \& Rivier 1984).

This study found that the vertices of cells of $P$. haitanensis could be used to fit ellipses with an average form deviation of $0.00 \pm 3.14 \%$ (8,291 vertices in 1375 cells were examined; Table 1; Dataset S2). We found similar results in the other 2D structures (Table 2; Dataset S3). Thus, the polygonal cells of 2D structures could be considered to be EIPs, which ensured that all of the cells were convex polygons. The ratios of the area of the cell and fitted EMIP $\left(\mathrm{A}_{C} / \mathrm{A}_{M I P}\right)$ of P. haitanensis ranged from 0.48 to 1.00 with an average value of $0.90 \pm 0.07$ (Table 1), and $90 \%$ of the values were concentrated in a range from 0.78 to 0.97 (Dataset S2). The random-seeded Voronoi diagrams and three kinds of epidermal tissues showed a similar average ratio of $\mathrm{A}_{C} / \mathrm{A}_{M I P}$. The $\mathrm{A}_{C}$ of amorphous $\mathrm{SiO}_{2}$, cross-sections of mollusk shells, and soap, however, were very close to $\mathrm{A}_{M I P}$ (Table 2; Dataset $\mathrm{S} 3$ ). Thus, we divided the $2 \mathrm{D}$ structures into three categories based on $\mathrm{A}_{C} / \mathrm{A}_{M I P}$ : Type I, monohedral tiling using six-edged EMIPs (e.g., tile by regular hexagons); Type II, tiling using different-size and different-edged EMIPs (e.g., 2D amorphous 
106

107

108

109

110

111

112

113

114

115

116

117

118

119

120

121

122

123

124

125

126

127

128

129

130

131

132

$\mathrm{SiO}_{2}$ ); and Type III, tiling using different-sized and different-edged EIPs. For amorphous $\mathrm{SiO}_{2}$, the bond length should be different to obey the ellipse packing. For the Types I and II 2D structures, $\mathrm{A}_{C}$ equals $\mathrm{A}_{M I P}$.

These results suggested that the fitted ellipse should be the smallest circumscribed ellipse of the polygonal cell, which was the reason we sought to find the smallest ellipse for four-edged cells in this study. A recent study reported similar phenomenon on single-celled microalga E. huxleyi (Xu et al. 2018). E. huxleyi cells were fully covered by interlocking calcite exoskeletons, and the specific geometry of exoskeletons resulted in the effective coverage area of exoskeletons tending to reach the maximal area of an inscribed polygon of ellipse-shaped exoskeletons.

Obviously, the effects of growth on cell topology for these three types of 2D structures were quite different. For 2D structures made of EMIPs, if the variations in topology were achieved by reconstruction or by transition to other types, the topological variations of all of the cells had to be finished synchronously to obey ellipse packing.

Otherwise, we observed a cell area less than $\mathrm{A}_{M I P}$. For example, the areas of polygonal cells were always equal to $A_{M I P}$ during the evolvement of soap (Table 2) (Aboav 1980). As for 2D structures made of different-sized and different-edged EIPs, most of the cells were smaller than their corresponding EMIPs. For biological 2D structures, complicated life activities strongly altered the cell size and topology (e.g., accumulation of organic components, respiration, cell division and fusion, water metabolism, and exposure of stressful conditions). Moreover, different metabolism rates between cells and the asynchronous cell cycle would make the dynamic behaviors of cell topology even more complicated. Based on geometric limits, Xu et al. (2018) proposed that the regular dodecahedron-shaped cells of coccolithophore Braarudosphaera spp. should be the resting or cyst stage of the life cycle. Similarly, the cells of living biological 2D structures should be EIPs rather than EMIPs, which suggested that living biological 2D structures belonged to Type III (Tables 1-2) and that specific cases may have manifested such that the complicated life activities would not influence the cell topology like the regular polyhedral cells of Braarudosphaera spp. The variations in the cell topology of Type III 2D structures had to be achieved by fine-tuning. In the following sections, we detail the effects of growth on cell topology for all three types of 2D structures.

The eccentric angle of neighboring vertices of a $n$-edged EMIP is equal to $2 \pi / n$ (Su 1987). Therefore, the eccentric angles of a six-edged EMIP equal to $60^{\circ}$ and the average internal angle is $120^{\circ}$. On the basis of observations of direction-specific divisions (which resulted in equal-sized divisions) and division-associated

Peer) reviewing PDF | (2019:01:34391:1:2:NEW 29 Mar 2019) 
133

134

136

137

138

140

141

142

143

144

145

146

147

direction changes of the cell edges (concentrated internal angles ranging from $100^{\circ}$ to $140^{\circ}$ ), $\mathrm{Xu}$ et al. (2017) found that $P$. haitanensis cells tended to form regular polygons. The closer the polygonal cell was to a regular polygon, the closer the cell was to a spherical shape, which could help maintain force balance (Chen 2008; Ingber et al. 2014).

Unbalanced forces could result in unequal-sized cell division (Kiyomitsu 2015). Equal-sized daughter cells, however, were always found in the cell proliferation of $P$. haitanensis thalli (Xu et al. 2017).

\section{Lewis's law}

Lewis's law is an empirical law that suggests that $\mathrm{A}_{C}$ of an $n$-edged cell is related linearly to $n$ (Chiu 1995; Lewis 1926; Lewis 1928; Weaire \& Rivier 1984). According to Eq. (2), the cell area of Type II 2D structures increased with edge number. To investigate the relationship between the number of edges and the cell area of Type III 2D structures, we used $P$. haitanensis thalli as the research material. The average values of $\mathrm{A}_{E}, \mathrm{~A}_{M I P}$, and $\mathrm{A}_{C}$ increased with $n$, whereas the difference between the average values of $\mathrm{A}_{E}$ and $\mathrm{A}_{C}$ decreased (Fig. 2A). Except for $n>8$, the average ratios of $a / b$ were stable regardless of the values of $n$ (Fig. 2B). Because $\mathrm{A}_{M I P}$ is $\frac{n}{2 \pi} \sin \left(\frac{2 \pi}{n}\right)$ times $\mathrm{A}_{E}(\mathrm{Su}$ 1987), the ratio of $A_{M I P} / A_{E}$ approaches one with an increase of $n$ (Fig. 2C). We found positive linear relationships between $\mathrm{A}_{C}$ and $\mathrm{A}_{E}\left(R^{2}=0.73, \mathrm{P}<0.0001\right.$; Fig. 2D) and between $\mathrm{A}_{C}$ and $\mathrm{A}_{M I P}\left(R^{2}=0.85, \mathrm{P}<0.0001\right.$, Fig. $\left.2 \mathrm{E}\right)$. Thus, $\mathrm{A}_{C}$ can be calculated by the following empirical equation:

$$
\mathrm{A}_{C}=0.80 \mathrm{~A}_{M I P}+78.79=0.40 \text { nabsin }\left(\frac{2 \pi}{n}\right)+78.79,
$$

where the maximal value of $n \sin \left(\frac{2 \pi}{n}\right)$ is

$$
\lim _{n \rightarrow \infty} n \sin \left(\frac{2 \pi}{n}\right)=2 \pi
$$

Because both $n \sin \left(\frac{2 \pi}{n}\right)$ and $\mathrm{A}_{E}$ increase with $n$ (Figs. 2A, 2B), $\mathrm{A}_{C}$ also increased with $n$, which was consistent with Lewis's law. Overall, the present study suggested that the relationship between $\mathrm{A}_{C}$ and $n$ is more complex than previous believed.

The cell proliferation of biological 2D structures can be used as a window to observe the dynamic behavior of cell topology during growth. By equal-sized division, mitosis shall strongly disturb cell topology. Obviously, division should separate a cell along the direction of the minor-axis of the fitted ellipse, making daughter cells closer to EMIPs (Fig. 3A). Nearly 150 years ago, Hofmeister proposed a similar idea called long-axis division 
159

160

161

162

163

164

165

166

167

168

169

170

171

172

173

174

175

176

177

178

179

180

181

182

183

184

185

(Hofmeister 1863). More complicated, however, Xu et al. (2017) found that divisions preferred to transect mother cells at midpoints of unconnected paired edges. Afterward, the direction of the cell edges were changed to concentrate the internal angles ranging from $100^{\circ}$ to $140^{\circ}$. Thus, the smallest number of edges per cell was four, and two equal-sized daughter cells were produced.

The ellipse packing is exactly a short-range order, which could influence both local and global cell topology. We used the average axes of the fitted ellipses and average number of edges to calculate the average variation on the internal angles (Table 1, Fig. 3A). Assuming an EMIP with six edges was divided along the minor axis of the ellipse, then ellipse packing should turn all three polygonal cells around the new vertex into EMIPs (Fig. 3B).

Thus, two daughter cells would be turned into two five-edged EMIPs with equal sizes, and the neighboring cell of the daughters would be turned into a seven-edged EMIP. The sum of the three angles around a vertex is $360^{\circ}$. Assuming the total disruptions on the three angles is kept to a minimum, on the basis of the least square method, the newly formed internal angle in the neighboring cell would be decreased from $180^{\circ}$ to $145.9^{\circ}$. This would explain the observation that the turning angle was $40 \pm 6^{\circ}$ (138 angles were examined) in the previous study by Xu et al. (2017). Meanwhile, those angles inherited from the mother cells also had to be adjusted to obey ellipse packing. Obviously, all of these changes on angles must be achieved by allometric growth of the cell edges. The long-axis division could help the cells retain their shapes closest to EMIPs. Finally, from a global perspective, the combined effect of ellipse packing and other short-range order (vertex coordination number is equal to three) turned all three angles around each vertex to $120^{\circ}$ (Fig. 3C). Overall, for biological 2D structures, ellipse packing placed restrictions on the direction of cell division and the turning angles of the cell edges.

\section{Aboav-Weaire's law}

If $m$ represents the average number of edges of cells surrounding an $n$-edged cell, then the relation between $m$ and $n$ of Type I 2D structures is $m=\mathrm{n}=6$. As for Type II and III 2D structures, we used Aboav-Weaire's law to describe the relation between $m$ and $n$ :

$$
m=(6-\beta)+\frac{6 \beta+\mu_{2}}{n},
$$

where six is the average number of cell edges of $2 \mathrm{D}$ structures, $\beta$ is a constant, and $\mu_{2}$ is related to the second moment of the edges of the $n$-edged cell (Weaire \& Rivier 1984). The present study and a previous study by Xu et

Peer) reviewing PDF | (2019:01:34391:1:2:NEW 29 Mar 2019) 
186

187

188

190

191

192

al. (2017) showed that all cells tended to form regular polygons, which indicated that the internal angles of a cell tended to be close to each other. According to Lewis's law, the cell area of Type II and III 2D structures increase with $n$. The average internal angle of an $n$-edged cell is $\pi-\frac{2 \pi}{n}$, which also increases with $n$. The sum of three angles around each vertex is $2 \pi$, which suggests that the average neighboring angle of the $n$-edged cell is decreasing with an increase of $n$. Consequently, the $m$, the average area, and the average internal angle of neighboring cells tend to decrease with an increase of $n$. Thus, Aboav-Weaire's law describes the representative level of a data set with $2 n$ neighboring angles in the total data set with $m n$ internal angles of the neighboring cells. In addition, the mean value of $m$ should also be equal to six.

On the basis of experimental studies, $\beta \approx 1.2$ was found to be conserved for several natural physical and biological structures(Aboav 1983; Aboav 1980; Mombach et al. 1990; Mombach et al. 1993). This number was very close to the average ratio of $a / b$ of cells of several kinds of 2D structures (Tables 1-2, Fig. 2B) and of the oval-shaped exoskeletons (faces) of E. huxleyi cells (Xu et al. 2018). In previous studies, $\mu_{2}$ has been assumed to be small (Edwards \& Pithia 1994; Lambert \& Weaire 1981). Regular hexagons could monohedrally tessellate a plane (Grünbaum \& Shephard 1987). This kind of tessellation also featured with ellipse packing and every vertex had a coordination number equal to three. This indicated that when $n=\langle n\rangle=6, \mu_{2}=0$, where $\langle n\rangle$ is the average number of cell edges. This study assumed

$$
\mu_{2}=\frac{6-n}{12}
$$

Thus, using Eq. (8), we have

$$
m=\left(6-\frac{a}{b}\right)+\frac{\frac{6 a}{b}+\frac{6-n}{12}}{n},
$$

where $a$ and $b$ are the semi-major axis and semi-minor axis of fitted ellipse of a $n$-edged cell, respectively. Then, Eq. (10) can be rewritten as follows:

$$
m=6+\frac{6-n}{n} \times\left(\frac{a}{b}+\frac{1}{12}\right)
$$

This equation could explain the relation between $m$ and $n$ of all three types of 2D structures. The calculated $m$ of cells of Types II and III 2D structures were very close to the real values by enumeration (Fig. 4A). The average difference between calculated $m$ and real $m$ was $-0.13 \pm 0.31$ (371 cells were examined). Because $\mu_{2}$ is very small, Aboav-Weaire's law could be approximately expressed as follows: 


$$
m \approx 6+\frac{6-n}{n} \times \frac{a}{b}
$$

213 The calculated $m$ using Eq. (11) and Eq. (12) showed only minor differences (Fig. 4A, supplementary raw data). In

214

addition, this study found an empirical relation for Type III 2D structures

$$
\frac{1}{12}=1-\frac{\mathrm{A}_{C}}{\mathrm{~A}_{M I P}}
$$

which can be expressed as follows

$$
\mathrm{A}_{C}=\mathrm{A}_{M I P}\left(1-\frac{1}{12}\right)=0.5 n a b \sin \left(\frac{2 \pi}{n}\right)\left(1-\frac{1}{12}\right) .
$$

The slope of the relationship between calculated $\mathrm{A}_{C}$ and measured $\mathrm{A}_{C}$ of cells of Types III 2D structures was very close to one $\left(R^{2}=0.98, \mathrm{P}<0.0001\right.$, Fig. $\left.4 \mathrm{~B}\right)$. The $a / b$ describes the deformation degree from circle to ellipse. Similarly, the present study proposed that, for Type III 2D structures, the number 1/12 describes the deformation degree from EMIP to EIP. Meanwhile, for Type III 2D structures, the Eq. (12) can be rewritten as follows:

$$
m=6+\frac{6-n}{n} \times\left(\frac{a}{b}+1-\frac{\mathrm{A}_{C}}{\mathrm{~A}_{M I P}}\right)
$$

\section{Variations of 2D topology}

We discussed the variations of cell topology of biological 2D structures in the previous sections. The randomseeded Voronoi diagrams are also Type III 2D structures, which were used to simulate the static structure of biological materials (Honda 1983; Sánchez-Gutiérrez et al. 2016). This study found that the ellipse packing creates a strong restriction on cellular geometry (e.g., the edge length and internal angle), which indicates that ellipse packing could be used to predict the effects of cell proliferation on cellular geometry. Thus, the combination of ellipse packing and Voronoi diagram may be applied to simulate the topological dynamic behaviors during the growth of biological 2D structures.

Although the average number of cell edges was always six, the distributions of the edge numbers showed big differences between the 2D materials and varied during growth (Aboav 1980; Aboav 1985; Büchner et al. 2016; Reich et al. 2018; Xu et al. 2017). Moreover, we know little about the relationships between the range of edge numbers and other topological parameters. In this study, we used the interval length (L) to describe the differences between the maximal and minimum edge numbers. Then, Type I 2D structures had the smallest $\mathrm{L}$ of 0 . 
237 During the evolvement of soap, L increased from 3 to 11 (Aboav 1980). Similar phenomena also have been

238

239

240

241

242

243

reported in physical 2D materials. For example, the point defects in hexagonal networks, one kind of local variations of topology, manifested with an increase of L from 0 to 2 (Büchner \& Heyde 2017).

To avoid confusion caused by the effects of observation scales, we discuss only the topological variations of a 2D physical material with constant mass, and these variations would not influence the connection pattern between atoms. Under the restrictions of ellipse packing and coordination number, the average number of cell edges was always six and the number of cells remained constant. We proposed five basic topological variations (Table 3):

V1. Reconstruction, which will not change the global topological parameters of the 2D structure but will create a new 2D structure with completely changed local topology. From a global scale, the area of the 2D structure will not be changed - for example, the destruction and rebuilding of graphene used the same number of carbon atoms.

V2. Scaling, which will not influence the type, $\mathrm{L}, n$, and $m$, but the area of the 2D structure and individual cells will be changed. The uniform scaling of ellipses has to be achieved by a uniform change in the edge lengths to maintain constant $a b$ and $a / b$.

V3. L-Variation of Type II, which was featured with a varied L of the Type II 2D structure and will not influence only the area of the 2D structure. Because we considered the Type I 2D structure to be a specific case of Type II with L equal to 0, the transition between Type I and Type II 2D structures actually belonged to LVariation. For 2D amorphous $\mathrm{SiO}_{2}$ film, the numbers of cell edges ranged from four to nine (Büchner \& Heyde 2017; Büchner et al. 2016), which indicated three intermediate states ( $\mathrm{L}=4,3$, and 2 ) occurred during the transition between crystalline (Type I) and amorphous (Type II) $\mathrm{SiO}_{2}$ film.

V4. Transition between Type II and Type III, which will change the area of 2D structure and individual cells. According to the new equation of Lewis's law in this study, the area of 2D structure should change by $1 / 12$ times, as should the volume if the height of 2D layers remained constant.

V5. Transition between Type I and Type III, which will change all parameters. The area of 2D structure changed by $1 / 12$ times.

Given the variations related to Types I and II 2D structures, the topological variations of all involved cells need to be synchronously finished to obey ellipse packing. The V2, V4, and V5 could influence the area of 2D

Peer) reviewing PDF | (2019:01:34391:1:2:NEW 29 Mar 2019) 
264

265

266

267

268

269

270

271

272

273

274

275

276

277

278

279

280

281

282

283

284

285

286

287

288

289

290

materials, which may be the most noticeable characteristic of these topological variations. The combination of these five basic topological variations would make it more difficult to understand the overall topological behavior, and the complexity of structure (e.g., heterogeneous materials, dimensionality of material) added further difficulties. For example, in the Voronoi diagrams with spiral lattice, the cells were arranged in a pattern of Fibonacci numbers (Rivier et al. 1984; Rivier et al. 2016). More work is needed to deeply understand the ellipse packing and its effects on global and local topology of 2D structures.

\section{D structures}

We considered every prismatic cell of the biological 2D structures to be a convex polyhedron with an average face number of eight. On the basis of a model study on 3D Voronoi froth with random seeds, if the coordination number of multi-polyhedral-celled 3D structures is four, then the average face number is $\left(\frac{48}{35}\right) \pi^{2}+2(\approx 15.54)$ (Meijering 1953; Weaire \& Rivier 1984). This number was very close to the average face number of 15.4 in the polyhedral cells of single-celled microalga $E$. huxleyi with a vertex coordination number of three (Xu et al. 2018). The difference in the average face number indicated that these 3D structures could not simplified as 2D structures. A convex polyhedral cell is a sealed 3D structure that has a positive curvature at every vertex and obeys Euler's law. Euler's law, however, does not set any restriction on six-edged faces (Grünbaum \& Motzkin 1963; Xu et al. 2018). This suggests that a given 3D structure does not necessarily need to be a sealed structure even it obeys Euler's law. The closure of polyhedra could be considered to be a basic level of uniform distribution of curvature. The face topology of polyhedra could be analyzed using software CaGe (Brinkmann et al. 2010).

Polygons with more than six edges induce locally negative curvature, and those with less than six edges induce positive curvature (Cortijo \& Vozmediano 2007). Thus, the polyhedral cells of E. huxleyi contained four-gons, five-gons, and six-gons, which helped maintain full coverage on the spherical surface (Xu et al. 2018). As for 2D tessellation using different-sized cells, the average edge number of six determined that the top and bottom faces of P. haitanensis cells contained four to ten edges (Table 1). Because of geometric limits, Lewis's law and AboavWeaire's law remained valid for the face topology of cells of E. huxleyi (Xu et al. 2018). For living biological 3D structures, as with the living biological 2D structures, growth influenced the topology of the polyhedral cells. Thus, on the basis of this study and the previous study by Xu et al. (2018), we suggested that the faces of the polyhedral 
291

292

293

294

295

296

297

cells would be EIPs rather than EMIPs to allow the cell topology to accommodate complicated life activities, which indicated that Lewis's law for Type III 2D structures (Eq. (15)) also may be applied to living biological 3D structures. Aboav-Weaire's law may be generalized to 3D structures with consideration for the distribution of curvature at vertices.

\section{Conclusion}

This study found that polygonal cells of natural and artificial 2D structures were inclined to form EMIPs. This phenomenon was named ellipse packing, which could be applied in simulations of the dynamics of cell topology during growth. We derived improved relations of Lewis's law and Aboav-Weaire's law and verified these findings using the semi-axes of fitted ellipses, cell area, and the number of cell edges. The present study suggested that Lewis's law and Aboav-Weaire's law are nonlinear relations, which mainly describe the effect of circle deformation on cell area and the edge number of neighboring cells. Ellipse packing determines the cell topology of 2D structures and growth patterns.

\section{Acknowledgments}

The author thanks Zhixue Chen and Fangyu Guo for their assistance collecting the geometric data of the cells. I thank my mother Yuntao Yan and my wife Huimin Cheng for their wholehearted support. We thank Accdon (www.accdon.com) for its linguistic assistance during the preparation of this manuscript.

\section{References}

Aboav D. 1983. The arrangement of cells in a net. II. Metallography 16:265-273.

Aboav DA. 1970. The arrangement of grains in a polycrystal. Metallography 3:383-390.

Aboav DA. 1980. The arrangement of cells in a net. Metallography 13:43-58.

Aboav DA. 1985. The arrangement of cells in a net. IV. Metallography 18:129-147.

Brinkmann G, Friedrichs OD, Lisken S, Peeters A, and Van Cleemput N. 2010. CaGe-a virtual environment for studying some special classes of plane graphs - an update. Match Communications In Mathematical And In Computer Chemistry 63:533-552.

Büchner C, and Heyde M. 2017. Two-dimensional silica opens new perspectives. Progress in Surface Science 92:341374. 10.1016/j.progsurf.2017.09.001

Büchner C, Liu L, Stuckenholz S, Burson KM, Lichtenstein L, Heyde M, Gao H-J, and Freund H-J. 2016. Building block analysis of 2D amorphous networks reveals medium range correlation. Journal of Non-Crystalline 
Solids 435:40-47. 10.1016/j.jnoncrysol.2015.12.020

Chen CS. 2008. Mechanotransduction - a field pulling together? Journal of Cell Science 121:3285-3292. $10.1242 /$ jcs. 023507

Chernov N, Huang Q, and Ma H. 2014. Fitting quadratic curves to data points. British Journal of Mathematics \& Computer Science 4:33-60.

Chiu S. 1995. Aboav-Weaire's and Lewis' laws-A review. Materials characterization 34:149-165.

Cortijo A, and Vozmediano MAH. 2007. Effects of topological defects and local curvature on the electronic properties of planar graphene. Nuclear Physics B 763:293-308. 10.1016/j.nuclphysb.2006.10.031

Edwards S, and Pithia K. 1994. A note on the Aboav-Weaire law. Physica A: Statistical Mechanics and its Applications 205:577-584.

Gibson MC, Patel AB, Nagpal R, and Perrimon N. 2006. The emergence of geometric order in proliferating metazoan epithelia. Nature 442:1038-1041.

Graustein W. 1931. On the average number of sides of polygons of a net. Annals of Mathematics:149-153.

Grünbaum B, and Motzkin TS. 1963. The number of hexagons and the simplicity of geodesics on certain polyhedra. Canadian Journal of Mathematics 15:744-751.

Grünbaum B, and Shephard GC. 1987. Tilings and patterns. The American Mathematical Monthly 95:xii+446.

Hofmeister W. 1863. Zusatze und Berichtigungen zu den 1851 veroffentlichen Untersuchungen der Entwicklung hoherer Kryptogamen. Jahrbucher für Wissenschaft und Botanik 3:259-193.

Honda H. 1983. Geometrical models for cells in tissues. 81:191-248. 10.1016/s0074-7696(08)62339-6

Ingber DE, Wang N, and Stamenovic D. 2014. Tensegrity, cellular biophysics, and the mechanics of living systems. Reports on Progress in Physics 77:046603. 10.1088/0034-4885/77/4/046603

Kiyomitsu T. 2015. Mechanisms of daughter cell-size control during cell division. Trends in Cell Biology 25:286-295.

Lambert C, and Weaire D. 1981. Theory of the arrangement of cells in a network. Metallography 14:307-318.

Lewis FT. 1926. The effect of cell division on the shape and size of hexagonal cells. The Anatomical Record 33:331355.

Lewis FT. 1928. The correlation between cell division and the shapes and sizes of prismatic cells in the epidermis of cucumis. The anatomical record 38:341-376.

Mason JK, Ehrenborg R, and Lazar EA. 2012. A geometric formulation of the law of Aboav-Weaire in two and three dimensions. Journal of Physics A: Mathematical and Theoretical 45:065001. 10.1088/17518113/45/6/065001

Meijering JL. 1953. Interface area, edge length, and number of vertices in crystal aggregates with random nucleation. Philips Res Rep.

Mombach J, Vasconcellos M, and de Almeida RM. 1990. Arrangement of cells in vegetable tissues. Journal of Physics D: Applied Physics 23:600.

Mombach JCM, de Almeida RM, and Iglesias JR. 1993. Mitosis and growth in biological tissues. Physical Review E 48:598.

Reich E, Schoeppler V, Lemanis R, Lakin E, Zolotoyabko E, Zollner D, and Zlotnikov I. 2018. Morphological and textural evolution of the prismatic ultrastructure in mollusc shells: A comparative study of Pinnidae species. Acta Biomater. 10.1016/j.actbio.2018.12.023

Rivier N, Occelli R, Pantaloni J, and Lissowski A. 1984. Structure of Bénard convection cells, phyllotaxis and crystallography in cylindrical symmetry. Journal de Physique 45:49-63. 10.1051/jphys:0198400450104900 
363

364

365

366

367

368

369

370

371

372

Rivier N, Sadoc JF, and Charvolin J. 2016. Phyllotaxis: a framework for foam topological evolution. Eur Phys $J E$ Soft Matter 39:7. 10.1140/epje/i2016-16007-8

Sánchez-Gutiérrez D, Tozluoglu M, Barry JD, Pascual A, Mao Y, and Escudero LM. 2016. Fundamental physical cellular constraints drive self-organization of tissues. EMBO Journal 35:77-88. 10.15252/embj.201592374

$\mathrm{Su} \mathrm{H.} \mathrm{1987.} \mathrm{The} \mathrm{characteristics} \mathrm{of} \mathrm{maximum} \mathrm{inscribed} \mathrm{and} \mathrm{minimum} \mathrm{circumscribed} \mathrm{polygons} \mathrm{of} \mathrm{ellipse.} \mathrm{Teaching}$ Mathematics 6:22-26.

Weaire D. 1974. Some remarks on the arrangement of grains in a polycrystal. Metallography 7:157-160.

Weaire D, and Rivier N. 1984. Soap, cells and statistics_random patterns in two dimensions. Contemporary Physics 25:59-99. 10.1080/00107518408210979

Xu K, Hutchins D, and Gao K. 2018. Coccolith arrangement follows Eulerian mathematics in the coccolithophore Emiliania huxleyi. PeerJ 6:e4608. 10.7717/peerj.4608

Xu K, Xu Y, Ji D, Chen T, Chen C, and Xie C. 2017. Cells tile a flat plane by controlling geometries during morphogenesis of Pyropia thalli. PeerJ 5:e3314. 10.7717/peerj.3314 
Figure 1

Geometry of polygonal cell and fitted ellipse.

(A) Coordinates of the vertices of a polygonal cell and fitted ellipse. We plotted the ellipse using software R plus package Conics (Chernov et al. 2014). (B) A diagram shows semimajor-axis (a), semi-minor-axis (b), angle $(\delta)$ between line VC and X-axis, angle $(\theta)$ of tilt of the major, distance $\left(D_{v c}\right)$ between the center of the ellipse and vertex of polygonal cell , distance $(R)$ from the center of the ellipse to the cross point of line VC and fitted ellipse.

A

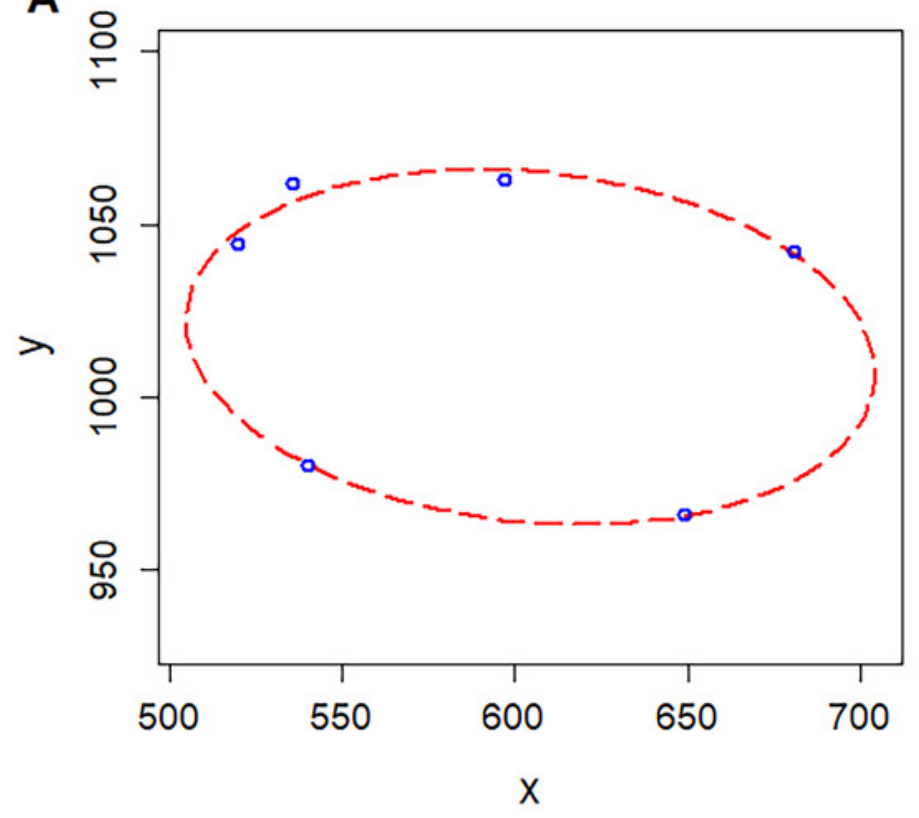

B

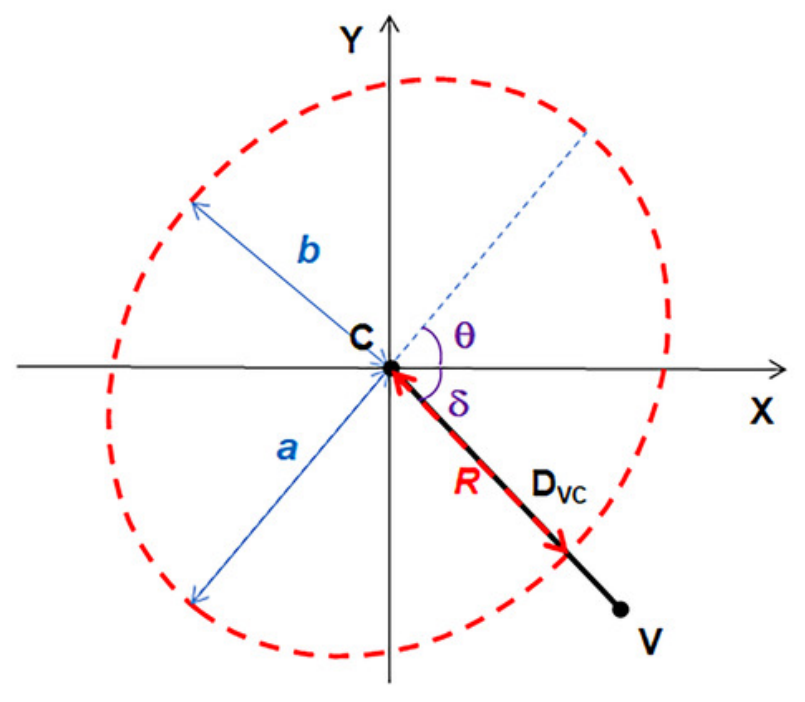




\section{Figure 2}

Relationships between $n, \mathrm{~A}_{C}, \mathrm{~A}_{M P}$, and $\mathrm{A}_{E}$ of $P$. haitanensis cells.

(A) Relationships between the number of cell edges $n$, area of cell $A_{c}$, area of the maximal inscribed polygon $A_{M P}$, and area of fitted ellipse $A_{E}$. Big symbols represent the average values of $A_{C}, A_{M P}$, and $A_{E}$, whereas small symbols represent the raw data (1375 cells were analyzed). (B) Relationship between $n$ and ratio of a/b. (C) Relationship between $n$ and ratio of $A_{M I P} / A_{E}$. (D) Relationship between $A_{C}$ and $A_{\text {MIP }}$ (1375 cells were analyzed). (E) Relationship between $A_{C}$ and $A_{E}$ (1375 cells were analyzed). 

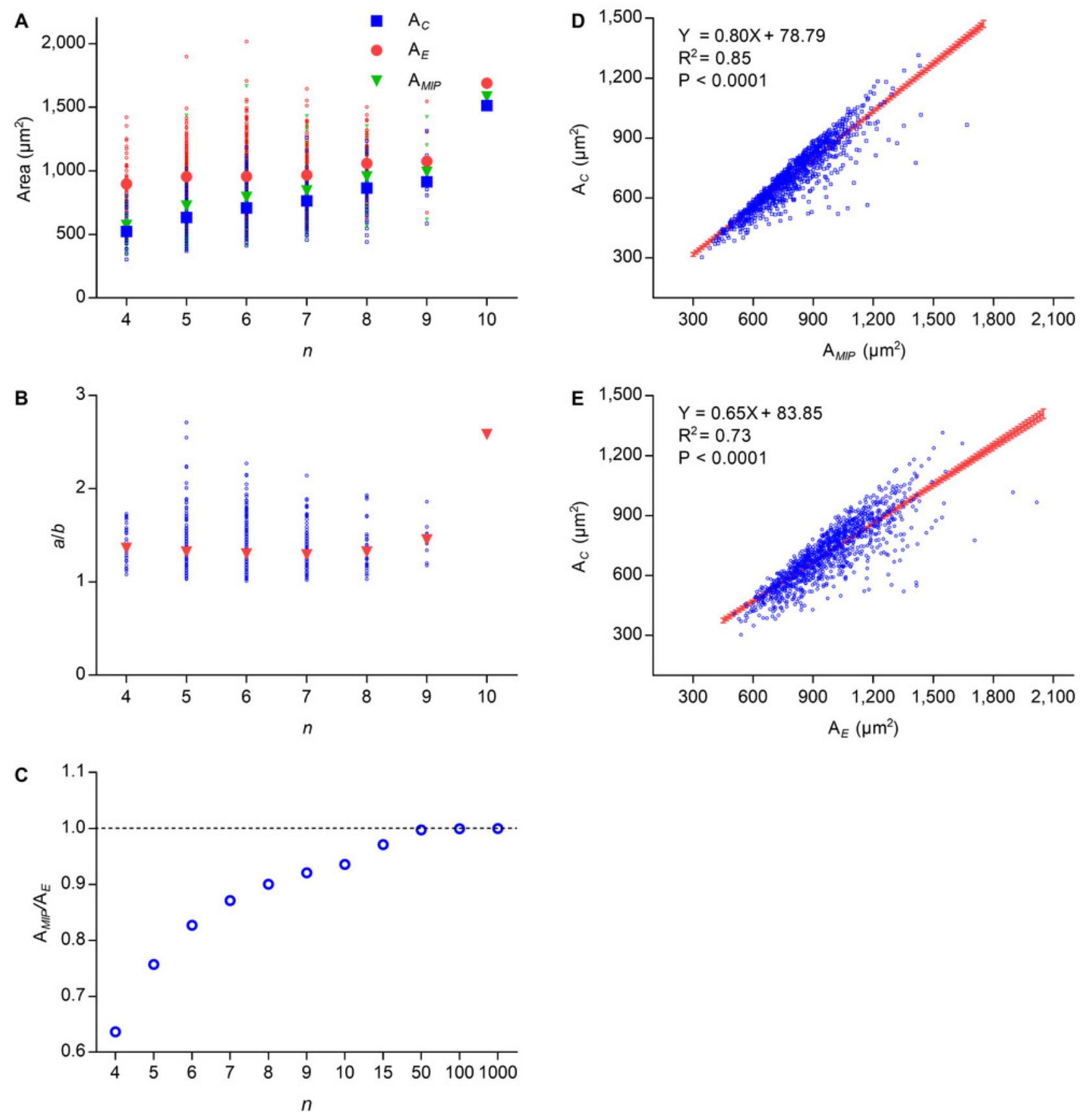


\section{Figure 3}

Cell division obeys ellipse packing.

(A) Red dashed lines represent that division of the maximally inscribed six-gon divided the cell along the minor axis of the ellipse and produced two equal-size daughters. Blue dashed line shows that an edge was separated by a new vertex, which produced three new angles (bottom). (B) Ellipse packing turned the two daughters into maximally inscribed five-gons (top left) by allometric growth of cell edges, whereas the neighboring seven-gon also turned into an EMIP (top right). To minimize the total disruption on the three angles, the turning angle in the neighboring cell should be $34.1^{\circ}$ (bottom). (C) The three angles around each vertex tended to be $120^{\circ}$. The ratios of $a / b$ of all of the ellipses were set to an average value of 1.3 (Table 1 ).

A

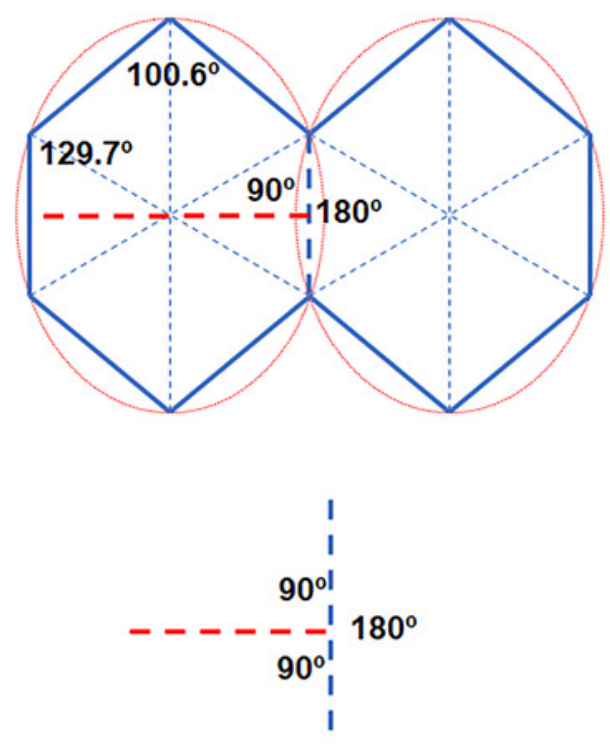

B

Local topology

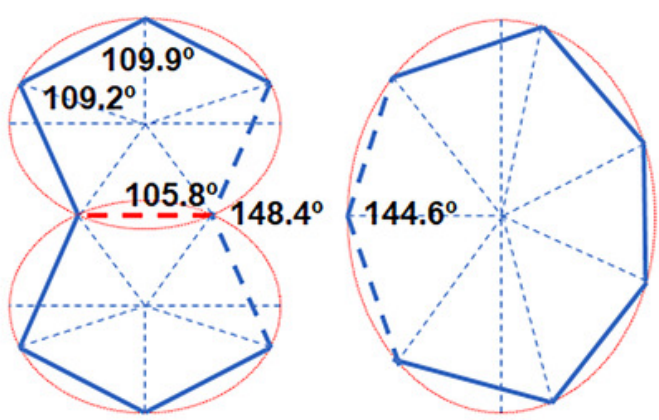

C Global topology 


\section{Figure 4}

Examinations of the relations of Lewis's law and Aboav-Weaire's law.

(A) Relationship between the real $m\left(m_{R}\right)$ and the calculated $m\left(m_{c}\right)$ of an n-edged cell (371 cells were examined). We used Eqs. (11) and (12) to calculate m. (B) Relationship between real and calculated area $\left(A_{c}\right)$ of an n-edged polygonal cell of all Type III 2D structures (1475 cells were examined). We used Eq. (14) to calculate $A_{c}$. The units of the cell area of $P$. haitanensis (1375 cells, Table 1) and the other Type III 2D structures (100 cells, Table 2) were $\mu \mathrm{m}^{2}$ and pixel ${ }^{2}$, respectively. 

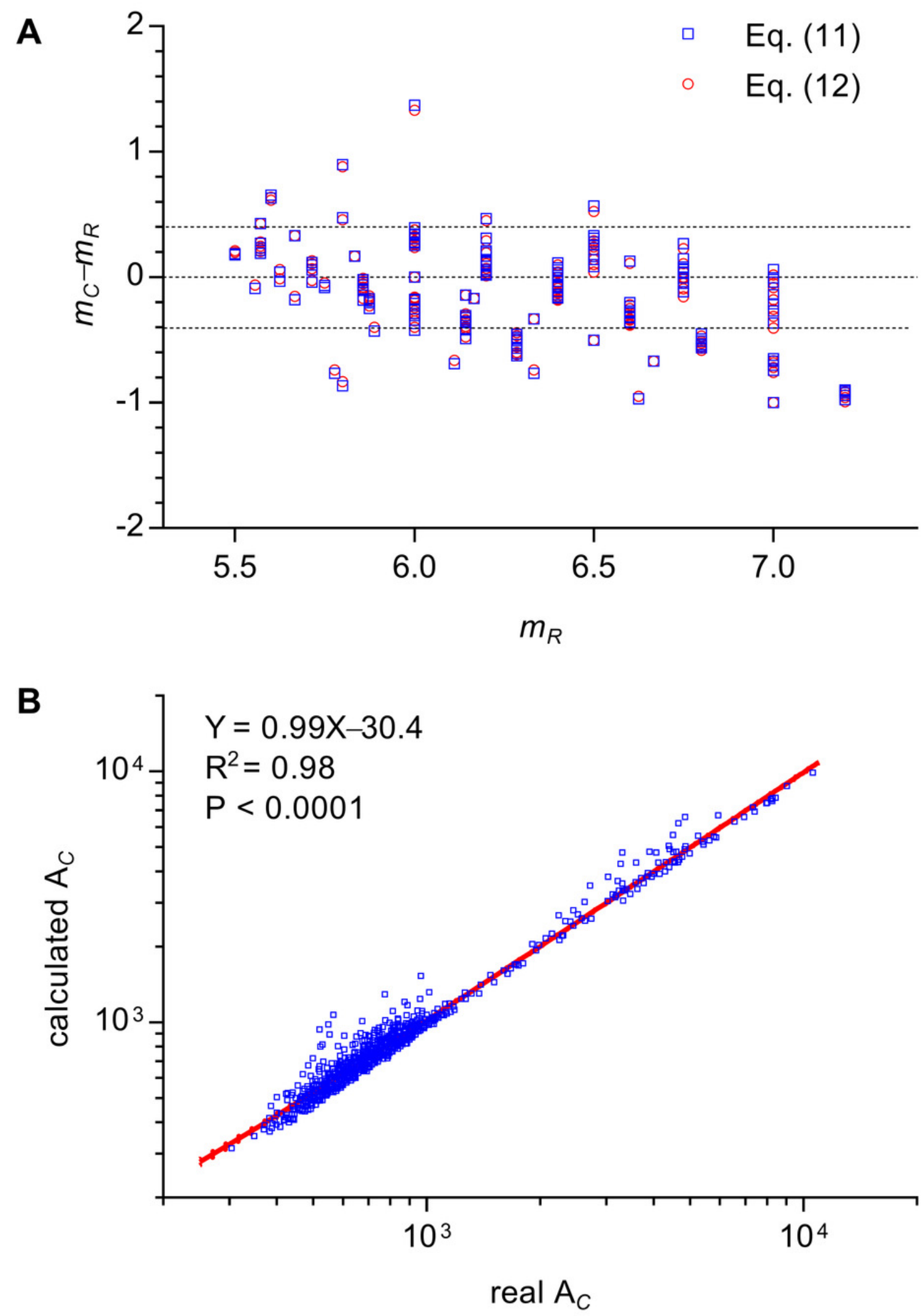


\section{Table $\mathbf{1}$ (on next page)}

Parameters of polygonal cells of $P$. haitanensis and fitted ellipses. 


\begin{tabular}{llll}
\hline & & & \\
\hline Parameters & Mean $\pm \mathrm{SD}$ & Range & Sample \\
& & & number \\
\hline Average number of cell edges & $6.03 \pm 0.88$ & $4-10$ & 1375 \\
Form deviation $(F D, \%)$ & $0.00 \pm 3.14$ & $-13.94-20.56$ & 8291 \\
Fitted semi-major-axis $(a, \mu \mathrm{m})$ & $19.86 \pm 2.76$ & $13.91-37.22$ & 1375 \\
Fitted semi-minor-axis $(b, \mu \mathrm{m})$ & $15.34 \pm 1.72$ & $9.29-21.76$ & 1375 \\
a/b & $1.31 \pm 0.21$ & $1.01-2.71$ & 1375 \\
Area of fitted ellipse $\left(\mathrm{A}_{E}, \mu \mathrm{m}^{2}\right)$ & $961.80 \pm 195.25$ & $506.16-2016.86$ & 1375 \\
Area of the maximal inscribed polygon & $788.19 \pm 172.00$ & $343.24-1667.93$ & 1375 \\
of fitted ellipse $\left(\mathrm{A}_{M I P}, \mu \mathrm{m}^{2}\right)$ & & & 1375 \\
Area of cell $\left(\mathrm{A}_{C}, \mu \mathrm{m}^{2}\right)$ & $705.98 \pm 148.98$ & $303.94-1512.63$ & 1375 \\
$\mathrm{~A}_{C} / \mathrm{A}_{M I P}$ & $0.90 \pm 0.07$ & $0.48-1.00$ & \\
\hline
\end{tabular}




\section{Table 2 (on next page)}

Parameters of polygonal cells of 2D structures.

Parameters $a / b, A_{C} / A_{M I P}$, and $F D$ represent the ratio of fitted semi-major-axis/semi-minoraxis, ratio of cell area/EMIP, and form deviation, respectively. Except for the last 2D structure, we derived the images of the others from published papers: amorphous $\mathrm{SiO}_{2}$ (Büchner et al. 2016), cross-sections of mollusk shells (Reich et al. 2018), soap (Aboav 1980), Voronoi diagrams (Aboav 1985), epidermal tissues of Agave attenuate , Allium cepa (onion), and Allium sativum (garlic) (Mombach et al. 1990). Sample numbers are shown in parentheses. 
1

\begin{tabular}{lllll}
\hline \multicolumn{1}{c}{ 2D structures } & $a / b$ & $\mathrm{~A}_{C} / \mathrm{A}_{M I P}$ & $F D(\%)$ \\
\hline \multirow{2}{*}{ Type II } & Amorphous $\mathrm{SiO}_{2}$ & $1.20 \pm 0.12(10)$ & $0.99 \pm 0.01(10)$ & $0.00 \pm 3.73(62)$ \\
& Cross-sections of mollusk shells & $1.14 \pm 0.07(30)$ & $0.97 \pm 0.02(30)$ & $0.00 \pm 0.89(170)$ \\
& Soap & $1.11 \pm 0.05(20)$ & $0.98 \pm 0.01(20)$ & $0.00 \pm 1.14(118)$ \\
\hline \multirow{2}{*}{ Type III } & $1.43 \pm 0.25(50)$ & $0.87 \pm 0.09(50)$ & $0.01 \pm 2.48(286)$ \\
& Allium cepa (onion) & $3.24 \pm 0.78(10)$ & $0.95 \pm 0.03(10)$ & $0.01 \pm 2.46(56)$ \\
& Allium sativum (garlic) & $3.43 \pm 1.03(10)$ & $0.92 \pm 0.03(10)$ & $0.03 \pm 4.58(57)$ \\
& Agave attenuat & $1.13 \pm 0.06(10)$ & $0.98 \pm 0.01(10)$ & $0.00 \pm 1.14(60)$ \\
& Allium cepa (onion) & $1.94 \pm 0.38(20)$ & $0.95 \pm 0.04(20)$ & $0.00 \pm 1.84(113)$ \\
\hline
\end{tabular}

2 


\section{Table 3 (on next page)}

Fivekinds of basic topology variations of 2D physical structures with constant mass.

V1: Reconstruction; V2: Scaling; V3: L-Variation; V4: Transition between Type II and Type III ; V5: Transition between Type I and Type III. Symbol ' represents the parameter will not be changed, and Ö represents the parameter will be changed. 


\begin{tabular}{|c|c|c|c|c|c|c|}
\hline & Parameters & V1 & $\mathrm{V} 2$ & $\mathrm{~V} 3$ & V4 & V5 \\
\hline \multirow{4}{*}{ Global } & Type of 2D structure & $x$ & $x$ & $\times$ or $\sqrt{ }$ & $\sqrt{ }$ & $\sqrt{ }$ \\
\hline & Area of 2D structure & $x$ & $\sqrt{ }$ & $x$ & $\sqrt{ }$ & $\sqrt{ }$ \\
\hline & Interval length of range of edge & & & & & \\
\hline & number (L) & $x$ & $x$ & $\sqrt{ }$ & $x$ & $\sqrt{ }$ \\
\hline \multirow{5}{*}{ Local } & Number of cell edges $(n)$ & $\sqrt{ }$ & $x$ & $\sqrt{ }$ & $x$ & $\sqrt{ }$ \\
\hline & Area of cell $\left(\mathrm{A}_{C}\right)$ & $\sqrt{ }$ & $\sqrt{ }$ & $\sqrt{ }$ & $\sqrt{ }$ & $\sqrt{ }$ \\
\hline & $a b$ & $\sqrt{ }$ & $\sqrt{ }$ & $\sqrt{ }$ & $x$ & $\sqrt{ }$ \\
\hline & $a / b$ & $\sqrt{ }$ & $\times$ or $\sqrt{ }$ & $\sqrt{ }$ & $x$ & $\sqrt{ }$ \\
\hline & $\begin{array}{l}\text { Average number of edges of } \\
\text { neighboring cells }(m)\end{array}$ & $\sqrt{ }$ & $x$ & $\sqrt{ }$ & $x$ & $\sqrt{ }$ \\
\hline
\end{tabular}

